\title{
Electromagnetohydrodynamic (EMHD) peristaltic flow of solid particles in a third-grade fluid with heat transfer
}

\author{
M.M. Bhatti ${ }^{1, a}$, A. Zeeshan ${ }^{2}$ And R. Ellahi ${ }^{2,3}$ \\ 1 Shanghai Institute of Applied Mathematics and Mechanics, Shanghai University Shanghai 200072, P.R. China \\ 2 Department of Mathematics and Statistics, International Islamic University, Islamabad, Pakistan \\ 3 Department of Mathematics, Faculty of Science, Taibah University, Madinah Munawwarah, Saudi Arabia
}

Received 22 May 2016, Accepted 29 Octobre 2016

\begin{abstract}
In this article, the Electromagnetohydrodynamic (EMHD) flow with heat transfer on thirdgrade fluid containing small particles has been investigated. The influence of thermal radiation are also taken under considerations. An external vertical magnetic field and horizontal electric field is applied while the induced magnetic field is very small and assumed to be negligible here. Long wavelength and zero Reynolds number approximation have been used to simplify the governing flow problem. The resulting highly nonlinear coupled ordinary differential equations are solved analytically with the help of perturbation method. The influence of all the pertinent parameters is discussed with the help of graphs for velocity profile, temperature profile, and pressure rise. Numerical computation has also been used to determine the expression for pressure rise. Trapping mechanism is also discussed with the help of streamlines.
\end{abstract}

Key words: EMHD / non-Newtonian fluid / Particle-fluid / Heat transfer / Thermal radiation

\section{Introduction}

During the recent decades, various studies that are associated with the flow in flexible channel/pipe grab the attention of different researchers due to its numerous applications in biomedical engineering and industry. Sinusoidal motion due to symmetrical expansion and contraction of smooth muscles in a human body that helps in transporting various biological fluids is known as peristalsis. This phenomenon can be observed in various parts of the human body such transport of urine from a kidney to the bladder, bile ducts, esophagus, transport of blood in extracorporeal circulation, cilia motion, motion of spermatozoa and vasomotion of small blood vessels, respectively. A number of electronic devices have been introduced in biomedical engineering that's work on the mechanism of peristalsis such as blood pump machines, finger pumps, heart-lung machine, roller pumps and dialysis machine etc. Due to its wide range of applications many theoretical and experimental investigations on peristaltic flow have been presented by various researchers. For instance, Nadeem et al. [1] investigated the peristaltic phenomena with non-Newtonian nano Williamson fluid model in a curved channel having compliant walls. Hayat et al. [2] studied the peristaltic flow under the effects of

${ }^{\text {a }}$ Corresponding author: muhammad09@shu.edu.cn slip using Carreau-Yasuda model in a curved channel. Abbasi et al. [3] studied the considered similar geometry [2] and analyzed the peristaltic flow with an EyringPowell fluid model. Ellahi et al. [4,5] investigated the three-dimensional peristaltic motion of non-Newtonian in a rectangular duct. Abbas et al. [6] considered the threedimensional hyperbolic tangent fluid motion induced by the peristaltic wave having compliant walls and obtained the series solution with the help of Homotopy perturbation method (HPM).

Peristaltic flow with heat transfer has also many important clinical applications. Especially, a bio-heat transfer is a hot topic of research during the recent years. In biomedical engineering, bio-heat transfer in different tissues of the human body is very important in thermoregulation and thermotherapy system. Heat transfer in a human body occurs due to conduction process in tissues, occurrence of metabolic heat etc. Some more applications involve vasodilation, dilution methodology to analyze blood flow and to destroy undesirable cancer tissues. Various authors investigated the peristaltic flow with heat transfer in various geometrical aspects with different kinds of biological fluids. For instance, Hayat and Noreen [7] considered the heat transfer effects on the peristaltic motion of fourth-grade fluid with an induced magnetic field. Ellahi et al. [8] studied the effects of heat and mass transfer on peristaltic flow through a non-uniform 


\section{Nomenclature}

\begin{tabular}{|c|c|}
\hline$\tilde{U}, \tilde{V}$ & Velocity components in fixed frame \\
\hline$\tilde{X}, \tilde{Y}$ & Cartesian coordinate axis in fixed frame \\
\hline$C$ & Volume fraction density \\
\hline$N_{r}$ & Radiation parameter \\
\hline$\tilde{q}$ & Embedding parameter \\
\hline$\tilde{P}$ & Pressure in fixed frame \\
\hline$\tilde{a}$ & Wave amplitude \\
\hline$k$ & Thermal conductivity \\
\hline$E_{c}$ & Eckert number \\
\hline$P_{r}$ & Prandtl number \\
\hline$\alpha_{1} \alpha_{2}$ & Material constant \\
\hline$\tilde{b}$ & Width of the channel \\
\hline$\tilde{c}$ & Wave velocity \\
\hline$c$ & Specific heat at constant volume \\
\hline$R e$ & Reynolds number \\
\hline$\beta_{1} \beta_{2} \beta_{3}$ & Material constant \\
\hline$B_{0}$ & Magnetic field \\
\hline$M$ & Hartmann number \\
\hline $\bar{T}$ & Transpose \\
\hline $\bar{k}$ & Mean Absorption coefficient \\
\hline$\tilde{t}$ & Time \\
\hline$T$ & Temperature \\
\hline$S$ & Drag force \\
\hline $\mathbf{A}_{1} \mathbf{A}_{2} \mathbf{A}_{3}$ & Kinematical tensor \\
\hline$\mu_{s}$ & Viscosity of the fluid \\
\hline$Q$ & Volume flow rate \\
\hline$E$ & Electric parameter \\
\hline \multicolumn{2}{|r|}{ Greek symbols } \\
\hline$\sigma$ & Electric conductivity of the fluid \\
\hline $\bar{\sigma}$ & Stefan-Boltzmann constant \\
\hline$\tau$ & Stress tensor \\
\hline$\Lambda$ & Dimensionless fluid parameter \\
\hline$\varpi_{T}$ & Thermal equilibrium time \\
\hline$\varpi_{v}$ & Relaxation time of the particle \\
\hline$\lambda$ & Wavelength \\
\hline$\mu_{s}$ & Viscosity of the fluid \\
\hline$\theta$ & Dimensionless temperature \\
\hline$\phi$ & Amplitude ratio \\
\hline$\rho$ & Fluid density \\
\hline \multicolumn{2}{|r|}{ Subscripts } \\
\hline$f$ & Fluid phase \\
\hline$p$ & Particulate phase \\
\hline
\end{tabular}

rectangular duct and presented the exact solution. Hina et al. [9] explored the influence of heat and mass transfer on pseudoplastic fluid induced by peristaltic wave through a curved channel have compliant walls. Rehman et al. [10] studied the peristaltic flow of non-Newtonian Jeffrey fluid in asymmetric channel with heat transfer. Akbar et al. [11] analyzed the simultaneous effects of slip and thermal on biviscosity fluid motion due to peristaltic wave with heat transfer. Saleem and Haider [12] investigated the creeping flow mechanism with heat and mass transfer on peristaltic motion of the non-Newtonian fluid.
The peristaltic flow of particle-fluid suspension has become a major topic from engineering and scientific point of view. Such type of analysis is very much helpful to understand different engineering problems such as fluidization, powder technology, and sedimentation in medicine (i.e. erythrocyte sedimentation). Hung and Brown [13] initially described experimentally the peristaltic motion of solid particles. Srivastava and Srivastava [14] analyzed mathematically peristaltic motion of the particle-fluid suspension. Misra and Pandey [15] examined the peristaltic axisymmetric flow of solid particles in a viscous fluid through a uniform cross section of the uniform flexible tube. Mekheimer et al. [16] analyzed the peristaltic motion of particle-fluid suspension in a uniform planar channel and obtained the series solution with the help of perturbation method. Nagarani and Sarojamma [17] considered the peristaltic motion of small solid particles in non-Newtonian power-law fluid model through a channel. Yao et al. [18] considered the peristaltic motion of solid particles in a viscous fluid through a uniform porous channel. Bhatti et al. [19] studied the simultaneous effects of slip and endoscope on peristaltic flow of Jeffrey fluid model and obtained the exact solutions.

On the other hand, Magnetohydrodynamics characteristics define the features of electrically conducting fluids. Magnetohydrodynamics is very important and significant in different and industrial and physiological processes. Examples include magnetic resonance imaging (MRI), hyperthermia, magnetic drug targeting, cancer therapy etc. The controlled applications having frequency pulsating fields and low intensity are helpful to change the attitude of tissues and cells. Magnetically naïve of Chyme is convinced from the heat originated by the ions or the magnetic field located in the Chyme. These magnets are helpful to heat inflammations, uterus, bowel (intestines) and ulceration. The influence of Magnetohydrodynamics and with heat transfer and thermal radiation also play an important role in a different industrial process. Various authors examined theoretically and experimental the effects of Magnetohydrodynamics on peristaltic flow. Hayat et al. [20] examined simultaneously the slip effects and Magnetohydrodynamics on third-grade fluid through a planar channel. Sinha et al. [21] studied the MHD peristaltic flow with heat transfer through an asymmetric channel. Hameed et al. [22] investigated the heat transfer and Magnetohydrodynamics on peristaltic flow of secondgrade fluid towards a vertical tube. Akram et al. [23] explored the effects of heat and mass transfer with an inclined magnetic field on peristaltically induced motion with various waveforms. Ellahi et al. [24] analyzed the influence of Magnetohydrodynamics on peristaltic flow of non-Newtonian Jeffrey fluid model through a porous rectangular duct. He obtained the exact solution and observed that velocity of the fluid decreases due to the greater influence of magnetic field. Recently, Hina [25] studied the effects of MHD and slip on peristaltic flow of Ree-Eyring Powell fluid with heat and mass transfer.

With the above analysis in mind, the main objective of present study is to analyze the effects of EMHD on 


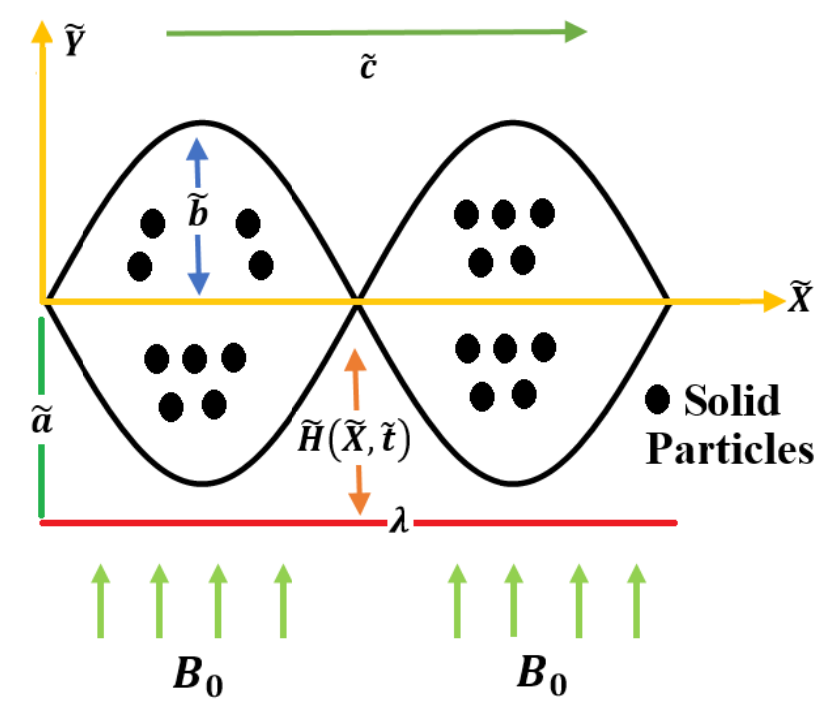

Fig. 1. Geometry of the problem.

peristaltically induced motion of solid particles in a thirdgrade with heat transfer through a uniform planar channel. The governing flow problem is modified with the help of long wavelength and low Reynolds number approximations. Analytical solutions have been obtained with the help of Homotopy perturbation method (HPM). The influence of all the governing parameters is discussed with the help of graphs. This paper is designed in the following way. Section 1 describes the introduction, Section 2 deals with the mathematical formulation of the problem. Section 3 shows the solution methodology and finally, Section 5 is devoted to graphical results and discussion.

\section{Mathematical Formulation}

Let us consider the Electromagnetohydrodynamic (EMHD) peristaltic flow of third grade fluid having small solid particles. We have taken Cartesian coordinate system in a two dimensional planar channel having $\tilde{X}$-axis considered along the channel and $\tilde{Y}$-axis taken normal to it (see Fig. 1). The fluid is electrically conducting by a vertical external magnetic field and the electric field is applied horizontally and the induced magnetic field is very low and considered to be zero here.

The geometry of the wall can be written as

$$
\tilde{H}(\tilde{X}, \tilde{t})=\tilde{a}+\tilde{b} \sin \frac{2 \pi}{\lambda}(\tilde{X}-\tilde{c} \tilde{t}) .
$$

The governing equation of continuity, momentum and thermal energy equation for fluid phase and particulate phase can be stated as

- Fluid Phase:

$$
\frac{\partial \tilde{U}_{f}}{\partial \tilde{X}}+\frac{\partial \tilde{V}_{f}}{\partial \tilde{Y}}=0
$$

$$
\begin{aligned}
& (1-C) \rho_{f}\left(\frac{\partial \tilde{U}_{f}}{\partial \tilde{t}}+\tilde{U}_{f} \frac{\partial \tilde{U}_{f}}{\partial \tilde{X}}+\tilde{V}_{f} \frac{\partial \tilde{U}_{f}}{\partial \tilde{Y}}\right)= \\
& -(1-C) \frac{\partial \tilde{P}}{\partial \tilde{X}}+(1-C)\left(\frac{\partial}{\partial \tilde{X}} \tau_{\tilde{X} \tilde{X}}+\frac{\partial}{\partial \tilde{Y}} \tau_{\tilde{X} \tilde{Y}}\right) \\
& +C S\left(\tilde{U}_{p}-\tilde{U}_{f}\right)-\sigma B_{0}^{2} \tilde{U}_{f}+\sigma B_{0} \hat{E} \\
& (1-C) \rho_{f}\left(\frac{\partial \tilde{V}_{f}}{\partial \tilde{t}}+\tilde{U}_{f} \frac{\partial \tilde{V}_{f}}{\partial \tilde{X}}+\tilde{V}_{f} \frac{\partial \tilde{V}_{f}}{\partial \tilde{Y}}\right)= \\
& -(1-C) \frac{\partial \tilde{P}}{\partial \tilde{Y}}+(1-C)\left(\frac{\partial}{\partial \tilde{X}} \tau_{\tilde{Y}} \tilde{X}+\frac{\partial}{\partial \tilde{Y}} \tau_{\tilde{Y} \tilde{Y}}\right) \\
& +C S\left(\tilde{V}_{p}-\tilde{V}_{f}\right)
\end{aligned}
$$

$$
\begin{aligned}
& (1-C) \rho_{f} c\left(\frac{\partial T_{f}}{\partial \tilde{t}}+\tilde{U}_{f} \frac{\partial T_{f}}{\partial \tilde{X}}+\tilde{V}_{f} \frac{\partial T_{f}}{\partial \tilde{Y}}\right) \\
= & k(1-C) \frac{\partial^{2} T_{f}}{\partial \tilde{Y}^{2}}+\frac{\rho_{p} c_{p} C}{\varpi_{T}}\left(T_{p}-T_{f}\right)+\frac{C S}{\varpi_{v}}\left(\tilde{U}_{f}-\tilde{U}_{p}\right)^{2} \\
+ & \mu_{s}(m 1-C) \tau_{\tilde{X} \tilde{Y}}\left(\frac{\partial \tilde{U}_{f}}{\partial \tilde{Y}}\right)+\sigma\left(\hat{E}-B_{0} \tilde{U}_{f}\right)^{2}-\frac{\partial Q_{R}}{\partial \tilde{Y}}
\end{aligned}
$$

- Particulate Phase:

$$
\frac{\partial \tilde{U}_{p}}{\partial \tilde{X}}+\frac{\partial \tilde{V}_{p}}{\partial \tilde{Y}}=0
$$

$$
\begin{aligned}
& C \rho_{p}\left(\frac{\partial \tilde{U}_{p}}{\partial \tilde{t}}+\tilde{U}_{p} \frac{\partial \tilde{U}_{p}}{\partial \tilde{X}}\right.\left.+\tilde{V}_{p} \frac{\partial \tilde{U}_{p}}{\partial \tilde{Y}}\right)= \\
&-C \frac{\partial \tilde{P}}{\partial \tilde{X}}+C S\left(\tilde{U}_{f}-\tilde{U}_{p}\right) \\
& C \rho_{p}\left(\frac{\partial \tilde{V}_{p}}{\partial \tilde{t}}+\tilde{U}_{p} \frac{\partial \tilde{V}_{p}}{\partial \tilde{X}}+\tilde{V}_{p} \frac{\partial \tilde{V}_{p}}{\partial \tilde{Y}}\right)= \\
&-C \frac{\partial \tilde{P}}{\partial \tilde{Y}}+C S\left(\tilde{V}_{f}-\tilde{V}_{p}\right)
\end{aligned}
$$

$$
\rho_{p} C c_{p}\left(\frac{\partial T_{p}}{\partial \tilde{t}}+\tilde{U}_{p} \frac{\partial T_{p}}{\partial \tilde{X}}+\tilde{V}_{p} \frac{\partial T_{p}}{\partial \tilde{Y}}\right)=\frac{\rho_{p} C c_{p}}{\varpi_{T}}\left(T_{f}-T_{p}\right)
$$

The mathematical expression for the drag coefficient and the empirical relation for the viscosity of the suspension can be defined as

$$
\begin{aligned}
S & =\frac{9 \mu_{0}}{2 \check{a}^{2}} \tilde{\lambda}(C) \tilde{\lambda}(C)=\frac{4+3 \sqrt{8 C-3 C^{2}}+3 C}{(2-3 C)^{2}} \\
\mu_{s} & =\frac{\mu_{0}}{1-\chi C}, \chi=0.07 e^{\left[2.49 C+\frac{1107}{T} e^{-1.69 C}\right]}
\end{aligned}
$$


The nonlinear radiative heat flux can be written as

$$
Q_{r}=-\frac{4 \bar{\sigma}}{3 \bar{k}} \frac{\partial T^{4}}{\partial \tilde{Y}}=-\frac{16 \bar{\sigma} T^{3}}{3 \bar{k}} \frac{\partial T}{\partial \tilde{Y}}
$$

The stress tensor of third grade fluid model is defined as $[26]$

$$
\begin{array}{r}
\tau_{i j}=\mu \mathbf{A}_{\mathbf{1}}+\alpha_{1} \mathbf{A}_{\mathbf{2}}+\alpha_{2} \mathbf{A}_{\mathbf{1}}^{\mathbf{2}}+\beta_{1} \mathbf{A}_{\mathbf{3}}+\beta_{2}\left(\mathbf{A}_{\mathbf{1}} \mathbf{A}_{\mathbf{2}}+\mathbf{A}_{\mathbf{2}} \mathbf{A}_{\mathbf{1}}\right) \\
+\beta_{3}\left(\operatorname{tr} \mathbf{A}_{\mathbf{1}}^{\mathbf{2}}\right) \mathbf{A}_{\mathbf{1}} .
\end{array}
$$

where

$$
\begin{gathered}
\mathbf{A}_{\mathbf{1}}=\operatorname{grad} \tilde{V}+(\operatorname{grad} \tilde{V})^{\bar{T}}, \mathbf{A}_{\mathbf{n}}=\frac{d \mathbf{A}_{m-1}}{d \tilde{t}} \\
+\mathbf{A}_{m-1}(\operatorname{grad} \tilde{V})+(\operatorname{grad} \tilde{V})^{\bar{T}} \mathbf{A}_{m-1} m=2,3 \ldots
\end{gathered}
$$

The respective boundary conditions are [17]

$$
\tilde{U}^{\prime}=0, T=T_{0}, a t \tilde{Y}=0, \tilde{U}=0, T=T_{1} a t \tilde{Y}=\tilde{H}
$$

Let us define the transformation variable from fixed frame to wave frame, we have

$$
\tilde{x}=\tilde{X}-\tilde{c} \tilde{t}, \tilde{y}=\tilde{Y}, \tilde{u}_{f, p}=\tilde{U}_{f, p}-\tilde{c}, \tilde{v}_{f, p}=\tilde{V}_{f, p}, \tilde{p}=\tilde{P}
$$

Introducing the following non-dimensional quantities

$$
\begin{aligned}
x & =\frac{\tilde{x}}{\lambda} y=\frac{\tilde{y}}{\tilde{a}} u_{f, p}=\frac{\tilde{u}_{f, p}}{\tilde{c}} P_{r}=\frac{\mu_{s} c}{k}, v_{f, p}=\frac{\tilde{v}_{f, p}}{\tilde{c} \delta}, \\
h & =\frac{\tilde{H}}{\tilde{a}}, \phi=\frac{\tilde{b}}{\tilde{a}}, p=\frac{\tilde{a}^{2}}{\lambda \tilde{c} \mu_{s}} \tilde{p}, R e=\frac{\rho \tilde{a} \tilde{c}}{\mu_{s}} \\
N_{1} & =\frac{S \tilde{a}^{2}}{\mu_{s}}, M=\sqrt{\frac{B_{0}^{2} \tilde{a}^{2} \sigma}{\mu_{s}}} \Gamma_{1}=\frac{\sigma}{(1-C) c\left(T_{1}-T_{0}\right)}, \\
\Gamma_{2} & =\frac{2 B_{0} \hat{E} c}{(1-C) c\left(T_{1}-T_{0}\right)} \theta_{f, p}=\frac{T_{f, p}-T_{0}}{T_{1}-T_{0}} \\
E_{c} & =\frac{\tilde{c}^{2}}{c\left(T_{1}-T_{0}\right)}, N_{r}=\frac{4 \bar{\sigma} T^{3}}{\mu_{s} c \bar{k}} E=\frac{\sigma B_{0} \tilde{a}^{2}}{\mu_{s}} \hat{E}
\end{aligned}
$$

Using Equations (15) and (16) in Equations (1) to (14), and considering the approximation of long wavelength and zero Reynolds number approximation. The resulting equations after some simplification for fluid phase can be written as

$$
\frac{1}{1-C} \frac{\mathrm{d} p}{\mathrm{~d} x}=\frac{\partial^{2} u_{f}}{\partial y^{2}}+3 \Lambda \frac{\partial^{2} u_{f}}{\partial y^{2}}\left(\frac{\partial u_{f}}{\partial y}\right)^{2}-M^{2}\left(u_{f}+1\right)+E
$$

$$
\begin{gathered}
\left(\frac{1}{P_{r}}+\frac{4}{3} N_{r}\right) \frac{\partial^{2} \theta_{f}}{\partial y^{2}}+E_{c}\left[\left(\frac{\partial u_{f}}{\partial y}\right)^{2}+\Lambda\left(\frac{\partial u_{f}}{\partial y}\right)^{4}\right] \\
+\frac{E_{c}}{N_{1}(1-C)}\left(\frac{\mathrm{d} p}{\mathrm{~d} x}\right)^{2}+M^{2}\left(u_{f}+1\right)^{2} E_{c} \\
-\Gamma_{2}\left(u_{f}+1\right)+\Gamma_{1}=0 .
\end{gathered}
$$

For particulate phase it can be written as

$$
\begin{aligned}
\frac{1}{N_{1}} \frac{\mathrm{d} p}{\mathrm{~d} x} & =\left(u_{f}-u_{p}\right), \\
\theta_{f} & =\theta_{p} .
\end{aligned}
$$

Their corresponding boundary conditions are

$$
u_{f}^{\prime}(0)=0, \theta_{f}(0)=0 \text { and } u_{f}(h)=-1, \theta_{f}(h)=1 .
$$

\section{Solution of the problem}

The exact solution for Equations (17) and (18) is not possible, hence we will employ Homotopy perturbation method (HPM) to obtain the solution of Equation (17) and (18). The Homotopy for the Equation (17) and (18) can be defined as

$$
\begin{aligned}
& H(w, \tilde{q})=(1-\tilde{q})\left(L(w)-L\left(\tilde{w}_{0}\right)\right) \\
& +\tilde{q}\left(L_{1}(w)+3 \Lambda \frac{\partial^{2} u_{f}}{\partial y^{2}}\left(\frac{\partial u_{f}}{\partial y}\right)^{2}-M^{2}\left(u_{f}+1\right)\right. \\
& \left.+E-\frac{1}{1-C} \frac{\mathrm{d} p}{\mathrm{~d} x}\right)
\end{aligned}
$$

$$
\begin{aligned}
& H(\Theta, \tilde{q})=(1-\tilde{q})\left(L(\Theta)-L\left(\tilde{\Theta}_{0}\right)\right) \\
& +\tilde{q}\left(L_{1}\left(\Theta_{f}\right)+E_{c}\left(\frac{\partial w_{f}}{\partial y}\right)^{2}+\frac{E_{c}}{N_{1}(1-C)}\left(\frac{\mathrm{d} p}{\mathrm{~d} x}\right)^{2}\right. \\
& \left.\quad+M^{2}\left(w_{f}+1\right)^{2} E_{c}-\Gamma_{2}\left(w_{f}+1\right)+\Gamma_{1}\right)
\end{aligned}
$$

The Linear operator $L$ is considered in the following form as

$$
L=\frac{\partial^{2}}{\partial y^{2}}
$$

The initial guess for the above linear operator is defined as

$$
\tilde{w}_{0}=-1+\left(y^{2}-h^{2}\right), \tilde{\Theta}_{0}=\frac{y}{h}
$$

Defining the following expansion

$$
\begin{aligned}
& w(y)=w_{0}(y)+\tilde{q} w_{1}(y)+\tilde{q}^{2} w_{2}(y)+\ldots \\
& \Theta(y)=\Theta_{0}(y)+\tilde{q} \Theta_{1}(y)+\tilde{q}^{2} \Theta_{2}(y)+\ldots
\end{aligned}
$$

Using Equations (26) and (27) in Equations (22) and (23) and comparing the powers of $\tilde{q}$, we get a system of linear differential equations with their relevant boundary conditions. According to methodology of Homotopy Perturbation method (HPM), we obtained the solution as $\tilde{q} \rightarrow 1$, we get

$$
\begin{aligned}
u_{f}(y) & =w(y)=w_{0}(y)+\tilde{q} w_{1}(y)+\tilde{q}^{2} w_{2}(y)+\ldots \\
\theta(r) & =\Theta(y)=\Theta_{0}(y)+\tilde{q} \Theta_{1}(y)+\tilde{q}^{2} \Theta_{2}(y)+\ldots
\end{aligned}
$$

The solution of velocity profile $\left(u_{f}, u_{p}\right)$ and temperature profile $\left(\theta_{f}, \theta_{p}\right)$ can be written in simplified form as 


$$
\begin{aligned}
u_{f}(y) & =-1+\left(y^{2}-h^{2}\right) \\
& +\frac{\left(h^{2}-y^{2}\right)\left(-6 E+6\left(-2+\frac{\mathrm{d} p}{\mathrm{~d} x}\right)+M^{2}\left(-5 h^{2}+y^{2}\right)+C\left(12+6 E+M^{2}\left(5 h^{2}-y^{2}\right)\right)\right)}{12(-1+C)} \\
& +\frac{24(1-C)\left(h^{4}-y^{4}\right) \Lambda}{12(1-C)}
\end{aligned}
$$

$$
\begin{aligned}
u_{p}(y) & =-\frac{1}{N_{1}} \frac{d p}{d x}-1+\left(y^{2}-h^{2}\right)+\frac{24(1-C)\left(h^{4}-y^{4}\right) \Lambda}{12(1-C)} \\
& +\frac{\left(h^{2}-y^{2}\right)\left(-6 E+6\left(-2+\frac{\mathrm{d} p}{\mathrm{~d} x}\right)+M^{2}\left(-5 h^{2}+y^{2}\right)+C\left(12+6 E+M^{2}\left(5 h^{2}-y^{2}\right)\right)\right)}{12(-1+C)},
\end{aligned}
$$

$$
\begin{aligned}
\theta_{f, p}(y) & =\frac{-5 k y\left(-6 h \Gamma_{1}+6 y \Gamma_{1}+5 h^{3} \Gamma_{2}-6 h^{2} y \Gamma_{2}+y^{3} \Gamma_{2}\right)}{60(1-C) N_{1}} \\
& +\frac{20 k y E_{c}(-1+C) h^{3} N_{1}-15 h d p / d x}{60(-1+C) N_{1}}-\frac{k^{2} y E_{c} h^{4} M^{2}}{2}+\frac{k y^{4} E_{c} h^{2} M^{2}}{6} \\
& +\frac{k y E_{c}\left(h^{5}\left(11 M^{2}+16 \Lambda\right)\right)}{30}+\frac{k y^{2} E_{c}\left(\left(15 \frac{d p}{d x}+(1-C) N_{1} y^{2}\left(10+y^{2}\left(M^{2}+16 \Lambda\right)\right)\right)\right)}{30(-1+C) N_{1}} .
\end{aligned}
$$

see equations (30)-(32) above.

The volume flow rate for fluid phase and particulate phase is given by

$$
Q=Q_{f}+Q_{p}
$$

Where

$$
\begin{aligned}
Q_{f} & =(1-C) \int_{0}^{h} u_{f} d y, \\
Q_{p} & =C \int_{0}^{h} u_{p} d y
\end{aligned}
$$

The pressure gradient $\mathrm{d} p / \mathrm{d} x$ is obtained after solving Equations (34) and (35). The non-dimensional pressure rise $(\Delta p)$ is evaluated numerically by using the following expression

$$
\Delta p=\int_{0}^{1} \frac{\mathrm{d} p}{\mathrm{~d} x} \mathrm{~d} x
$$

The expression for stream function is defined as

$$
u_{f, p}=\frac{\partial \Phi}{\partial y} v_{f, p}=-\frac{\partial \Phi}{\partial x}
$$

where

$$
k=\frac{P_{r}}{1+\frac{4}{3} P_{r} N_{r}}, h=1+\phi \sin 2 \pi x
$$

\section{Results and discussion}

In this section, the graphical results of different parameters are sketched with the help of computational software Mathematica. To analyse the novelties of different parameters Figures 2 to 13 are plotted against Hartmann number, Electric parameter, Prandtl number, Particle volume fraction, radiation parameter, Eckert number and Fluid parameter, respectively. In particular, we observed the effects of involved parameters on velocity profile, temperature profile and pressure rise. Streamlines are also plotted against all the physical parameters arise in the governing flow problem.

Figures 2 and 3 shows the velocity curves for fluid phase against particle volume fraction $\mathbf{C}$, Hartman number $\mathbf{M}$ Electric parameter $\mathbf{E}$ and fluid parameter $\boldsymbol{\Lambda}$. It depicts from Figure 2 that when the influence of particle volume fraction $\mathbf{C}$ is greater than it tends to oppose the flow and hence, the velocity of the fluid decreases. Moreover, we can see that when the magnetic field is getting higher than it does not provide any resistance to the flow and the velocity of the fluid increases. It can be observed from Figure 3 that when the fluid reveals Newtonian behaviour $(\boldsymbol{\Lambda}=0)$ then the magnitude of the velocity reduces whereas when the fluid shows non-Newtonian behaviour $(\boldsymbol{\Lambda} \neq \mathbf{0})$ then the velocity of the fluid rises. Furthermore, greater values of Electric parameter $\mathbf{E}$ boost the velocity of the fluid.

Figures 4 to 7 are sketched for temperature profile against different values of Prandtl number $\mathbf{P}_{\mathbf{r}}$, radiation parameter $\mathbf{N}_{\mathbf{r}}$, Particle volume fraction $\mathbf{C}$, Hartman number $\mathbf{M}$ and Electric parameter E. From Figure 4 we can see that Prandtl number $\mathbf{P}_{\mathbf{r}}$ enhances the temperature profile. In different heat transfer problems Prandtl number is very much favourable to handle the thickness of thermal boundary layer and momentum boundary layer. Moreover, we can analyse here that when the Prandtl number increases $\left(\mathbf{P}_{\mathbf{r}}>\mathbf{1}\right)$ then the thermal diffusivity 


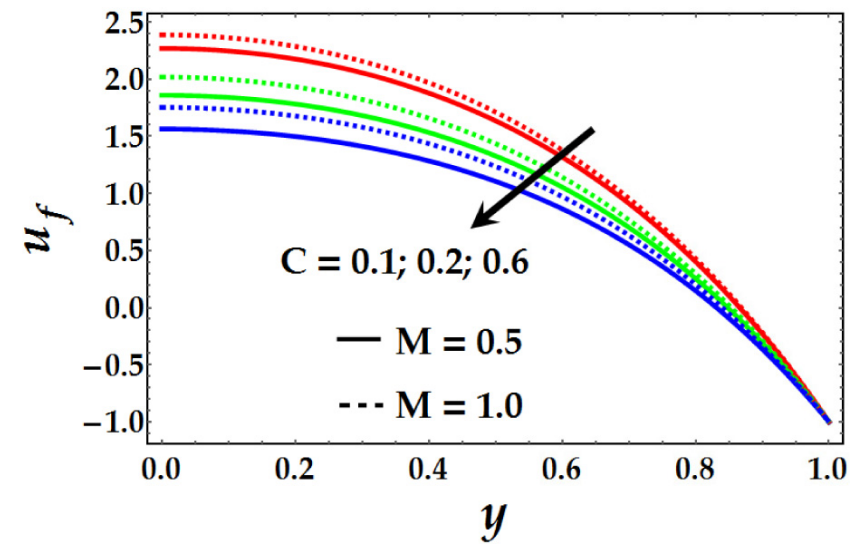

Fig. 2. Velocity profile for various values of $\mathbf{C}$ and $\mathbf{M}$ when $\phi=0.7, \mathrm{E}=0.5, \Lambda=0.5$.

is more dominating as compared to thermal diffusivity. We can also observe from this figure that in the absence of thermal radiation, the magnitude of the temperature profile is very large, however, in the presence of thermal radiation the temperature profile reduces significantly. It can be noticed from Figure 5 that when the particle volume fraction $\mathbf{C}$ and Hartmann number $\mathbf{M}$ increase then it tends to reduce the temperature profile. It can be observed from Figure 6 when the fluid shows non-Newtonian behaviour $(\boldsymbol{\Lambda} \neq \mathbf{0})$ then the temperature profile diminished. However, when the Electric parameter $E$ increases then it causes reduction in the temperature profile. Figure 7 shows the variation of Eckert number $\mathbf{E}_{\mathbf{c}}$ on temperature profile. We can see from this figure that Eckert number $\mathbf{E}_{\mathbf{c}}$ enhances very significantly the temperature profile, however temperature profile also increases when the parameter $\boldsymbol{\Gamma}_{\mathbf{2}}$ increases.

Figures 8 and 9 show the peristaltic pumping mechanism versus volume flow rate $\mathbf{Q}$ against various values of Hartman number $\mathbf{M}$, particle volume fraction $\mathbf{C}$, Electric parameter $\mathbf{E}$ and fluid parameter $\boldsymbol{\Lambda}$. Peristaltic pumping is very important phenomenon in human body that is very much helpful in transporting various biological fluids in a human body. From Figure 8 we can see that when the particle volume fraction and the flow rate are negative $(\mathbf{Q}<\mathbf{0})$ then pumping rate decreases very rapidly but when the flow rate are positive $(\mathbf{Q}>\mathbf{0})$ then the pumping rate boostes very quickly. Here, we can also see that when the influence of magnetic field is greater than it tends to rise the pressure rise. It depicts from Figure 9 that for higher values of Electric parameter leads to rise the pumping rate and similar behaviour has been observed when the fluid parameter $\boldsymbol{\Lambda}$ increases.

The next most important and engrossing part of peristaltic phenomena is trapping which can easily be analysed with the help of stream lines. Generally, the pattern of stream lines is similar to boundary wall in wave frame of reference. It is basically the generation of internally moving bolus. Some of the streamlines enclose and split a bolus under various conditions and this type of bolus maneuvers completely with peristaltic wave. Such type of

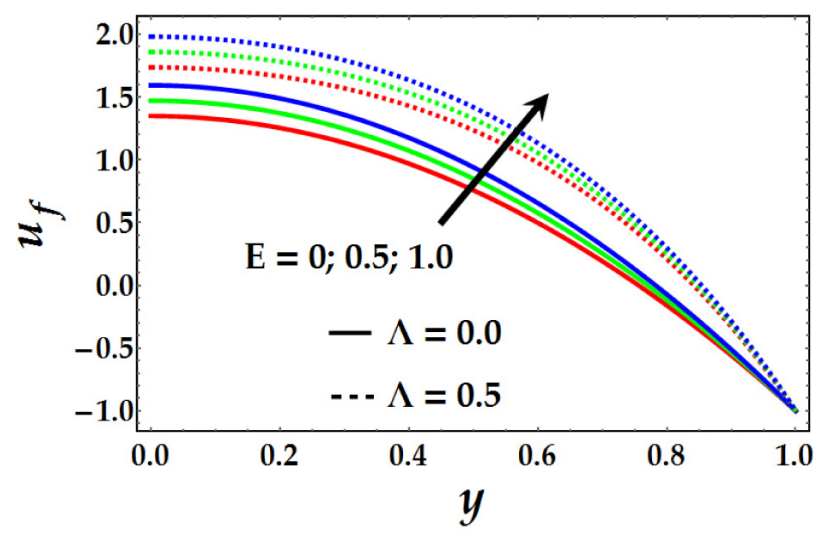

Fig. 3. Velocity profile for various values of $\mathbf{E}$ and $\boldsymbol{\Lambda}$ when $\phi=0.7, \mathrm{M}=0.5, \mathrm{C}=0.2$.

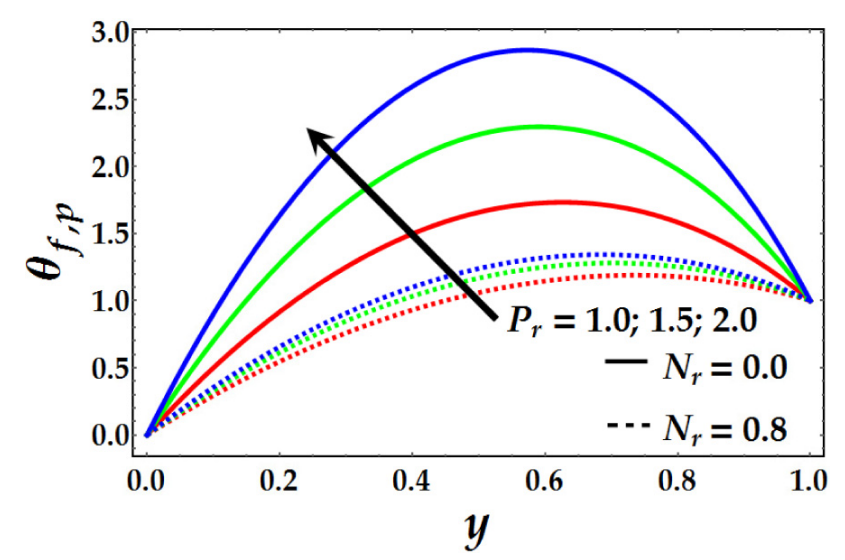

Fig. 4. Temperature profile for various values of $\mathbf{P}_{\mathbf{r}}$ and $\mathrm{N}_{\mathrm{r}}$ when $\phi=0.7, \mathrm{M}=\mathbf{0 . 5}, \mathrm{C}=\mathbf{0 . 2}, \mathbf{E}=\mathbf{0 . 5}, \mathrm{E}_{\mathrm{c}}=\mathbf{0 . 5}, \Lambda=$ 0.5 .

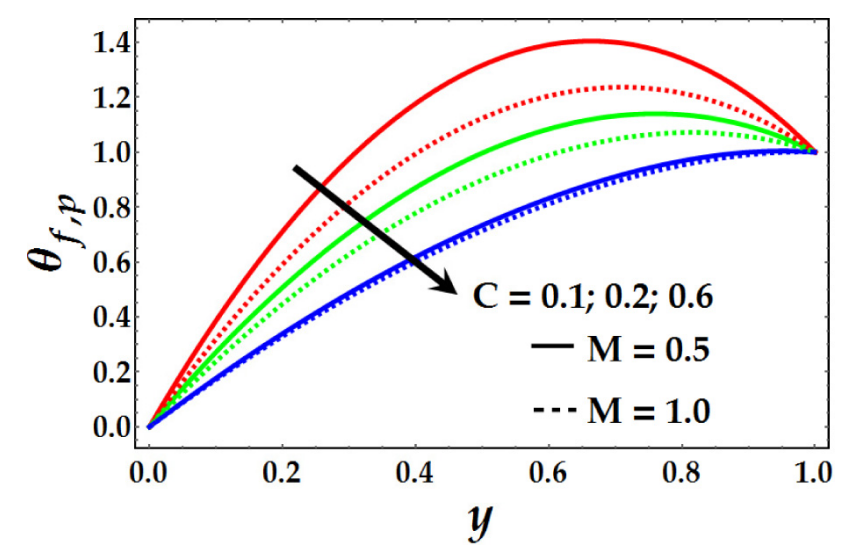

Fig. 5. Temperature profile for various values of $\mathbf{C}$ and $\mathbf{M}$ when $\phi=0.7, \mathrm{E}=0.5, \mathrm{E}_{\mathrm{c}}=0.5, \mathrm{P}_{\mathrm{r}}=1, \mathrm{~N}_{\mathrm{r}}=1 \Lambda=0.5$.

mechanism is known as trapping. It can be noticed from Figure 10 that when the particle volume fraction $\mathbf{C}$ increases then the magnitude of the bolus reduces whereas the number of bolus increases. From Figure 11 we can see that when the Electric parameter $\mathbf{E}$ increases then the size of the bolus increases very slowly while it doesn't cause any influence on the number of bolus. Figure 12 shows 


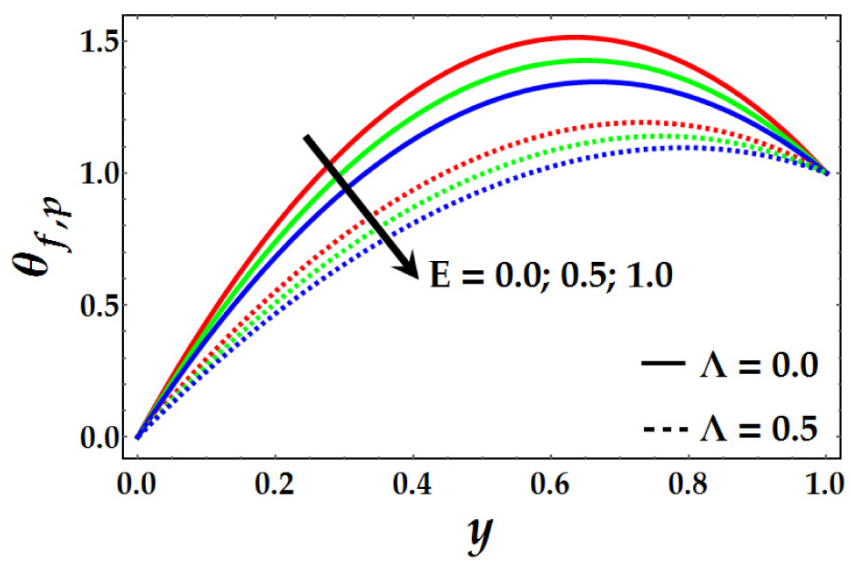

Fig. 6. Temperature profile for various values of $\mathbf{E}$ and $\boldsymbol{\Lambda}$ when $\phi=0.7, \mathrm{M}=0.5, \mathrm{C}=0.2, \mathrm{E}_{\mathrm{c}}=0.5, \mathrm{P}_{\mathrm{r}}=1, \mathrm{~N}_{\mathrm{r}}=1$.

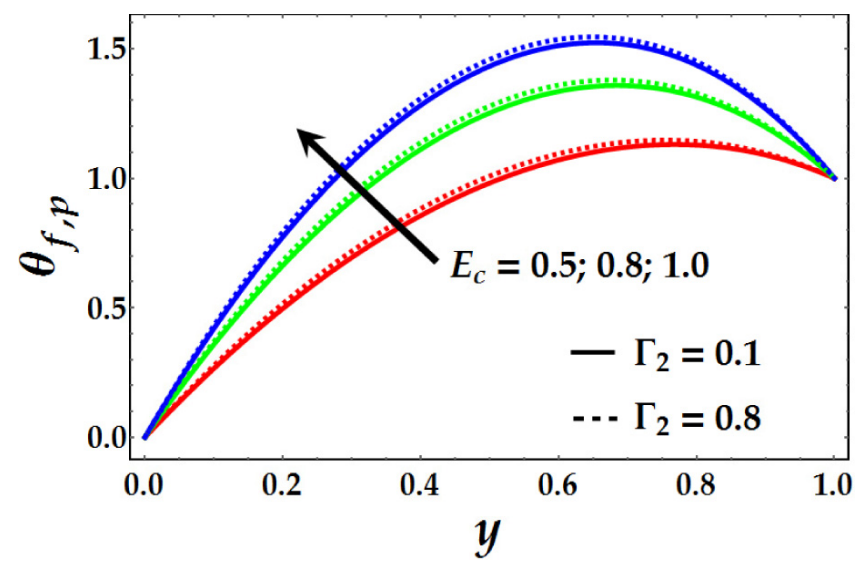

Fig. 7. Temperature profile for various values of $\mathbf{E}_{\mathbf{c}}$ and $\Gamma_{2}$ when $\phi=0.7, M=0.5, C=0.2, E=0.5, P_{r}=1, N_{r}=$ $\mathbf{1 \Lambda}=\mathbf{0 . 5}$.

that due to greater influence of magnetic field, then the trapping bolus does not change, however the size of the bolus increases. It can be visualized from Figure 13 that for large values of fluid parameter $\boldsymbol{\Lambda}$, the size of the bolus decreases very quickly and the number of bolus also reduces.

\section{Conclusion}

The present study describes the Electromagnetohydrodynamic (EMHD) effects on peristaltic flow of solid particles in a third grade-fluid with heat transfer through a uniform planar channel. The impact of thermal radiation is also taken into present study. The governing flow problem is simplified by neglecting the inertial forces and taking long wavelength approximation. The obtained highly nonlinear coupled partial differential equations are solved analytically with the help of Homotopy perturbation method (HPM). The major outcomes of the present study are summarized below: The effect of magnetic field and electric parameter enhances the velocity profile.

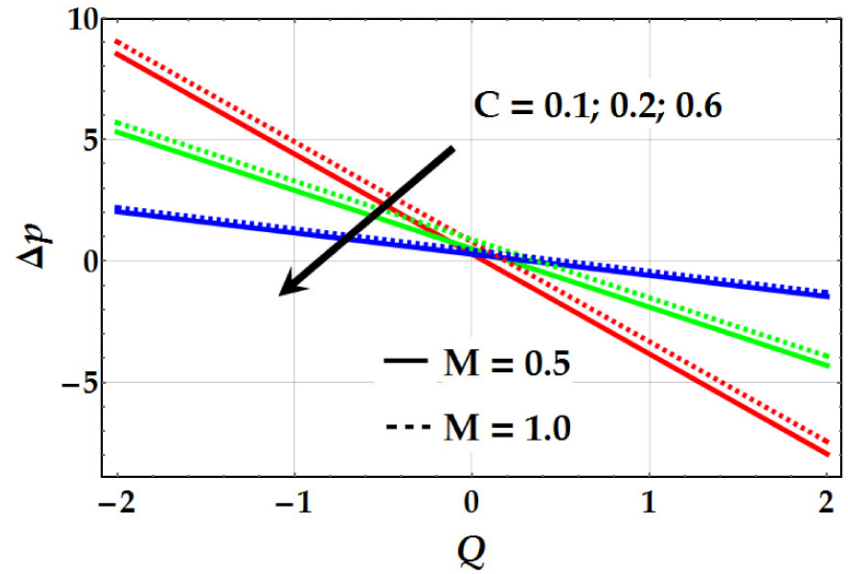

Fig. 8. Pressure rise vs. volume flow rate for various values of $\mathbf{C}$ and $\mathrm{M}$ when $\phi=\mathbf{0 . 7}, \mathbf{E}=\mathbf{0 . 5}, \boldsymbol{\Lambda}=\mathbf{0 . 5}$.

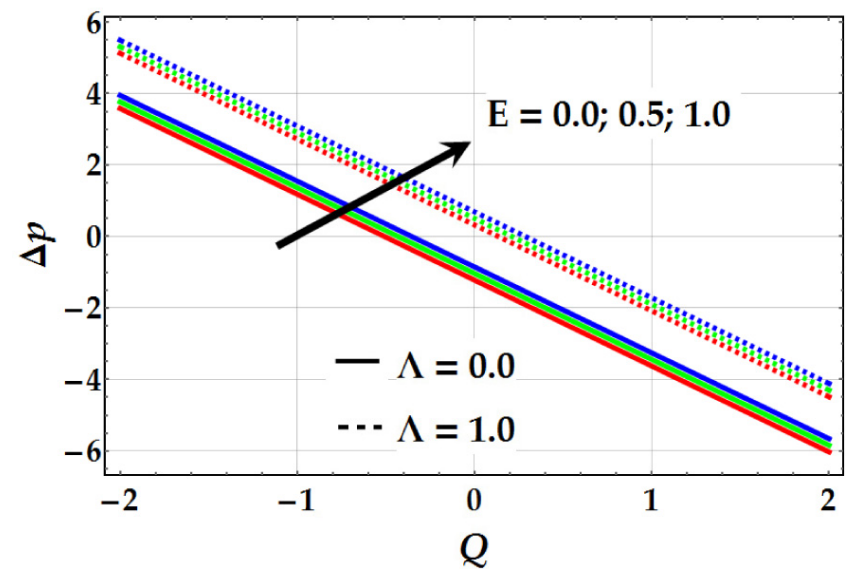

Fig. 9. Pressure rise vs. volume flow rate for various values of $\mathbf{E}$ and $\boldsymbol{\Lambda}$ when $\phi=\mathbf{0 . 7}, \mathbf{M}=\mathbf{0 . 5}, \mathbf{C}=\mathbf{0 . 2}$.
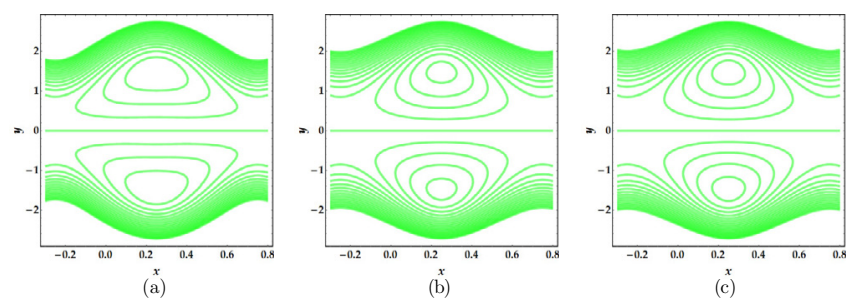

Fig. 10. Streamlines for various values of C, (a) 01, (b) 03, (c) 06, when $\phi=\mathbf{0 . 7}, \mathbf{M}=\mathbf{0 . 5}, \mathbf{E}=\mathbf{0 . 5}, \boldsymbol{\Lambda}=\mathbf{0 5}$.
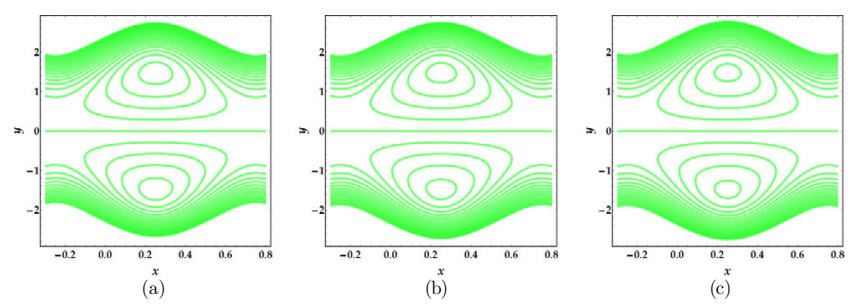

Fig. 11. Streamlines for various values of $\mathbf{E}$, (a) 0 , (b) 1 , (c) 2 , when $\phi=\mathbf{0 . 7}, \mathbf{M}=\mathbf{0 . 5}, \mathbf{C}=\mathbf{0 . 2}, \boldsymbol{\Lambda}=\mathbf{0 . 5}$. 

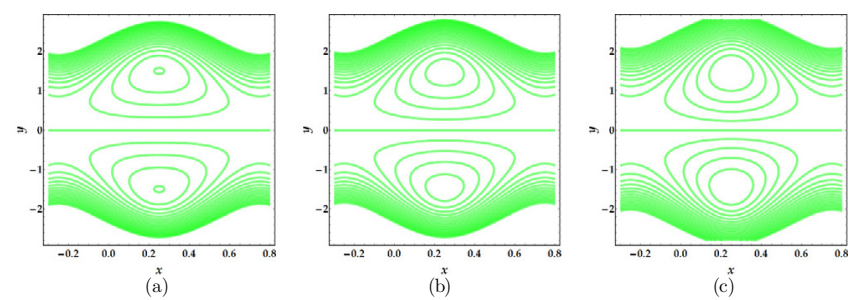

Fig. 12. Streamlines for various values of $\mathbf{M}$, (a) 0 , (b) 1 , (c) 2 , when $\phi=\mathbf{0 . 7}, \mathbf{C}=\mathbf{0 . 2}, \mathbf{E}=\mathbf{0 . 5}, \boldsymbol{\Lambda}=\mathbf{0 . 5}$.

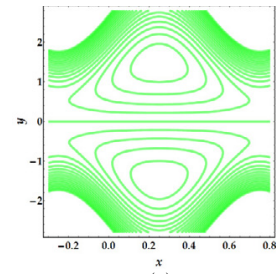

(a)

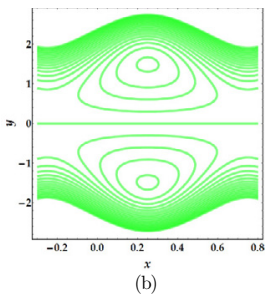

(b)

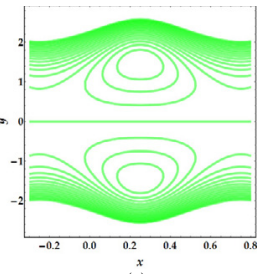

(c)
Fig. 13. Streamlines for various values of $\boldsymbol{\Lambda}$, (a) 0 , (b) 0.5 , (c) 2 , when $\phi=\mathbf{0 . 7}, \mathbf{M}=\mathbf{0 . 5}, \mathbf{C}=\mathbf{0 . 2}, \mathbf{E}=\mathbf{0 . 5}$.

- When the fluid depicts non-Newtonian behaviour then the velocity of the fluid rises.

- The impact of thermal radiation creates a reduction in the temperature profile.

- Temperature profile also decreases due to the influence of magnetic field and electric parameter.

- Electric field and fluid parameter enhance the pumping rate.

- The present results can also be obtained for Newtonian fluid by taking $\boldsymbol{\Lambda}=\mathbf{0}$ as a special case of our study.

\section{References}

[1] S. Nadeem, E.N. Maraj, N.S. Akbar, Investigation of peristaltic flow of Williamson nanofluid in a curved channel with compliant walls, Appl. Nanoscience 4 (2014) 511521

[2] T. Hayat, F.M. Abbasi, B. Ahmad, A. Alsaedi, Peristaltic transport of Carreau-Yasuda fluid in a curved channel with slip effects, PloS one 9 (2014) e95070

[3] F.M. Abbasi, A. Alsaedi, T. Hayat, Peristaltic transport of Eyring-Powell fluid in a curved channel, J. Aerospace Eng. 27 (2014) 04014037

[4] R. Ellahi, A. Riaz, S. Nadeem, Three dimensional peristaltic flow of Williamson fluid in a rectangular duct, Indian J. Phys. 87 (2013) 1275-1281

[5] R. Ellahi, A. Riaz, S. Nadeem, Three-dimensional peristaltic flow of a Williamson fluid in a rectangular channel having compliant walls, J. Mecha. Medicine Biol. 14 (2014) 1450002

[6] M.A. Abbas, Y.Q. Bai, M.M. Bhatti, M.M. Rashidi, Three dimensional peristaltic flow of hyperbolic tangent fluid in non-uniform channel having flexible walls, Alexandria Eng. J. 55 (2015) 653-662
[7] T. Hayat, S. Noreen, Peristaltic transport of fourth grade fluid with heat transfer and induced magnetic field, Comptes Rendus Mécanique 338 (2010) 518-528

[8] R. Ellahi, M.M. Bhatti, K. Vafai, Effects of heat and mass transfer on peristaltic flow in a non-uniform rectangular duct, Int. J. Heat Mass Transf. 71 (2014) 706-719

[9] S. Hina, M. Mustafa, T. Hayat, N.D. Alotaibi, On peristaltic motion of pseudoplastic fluid in a curved channel with heat/mass transfer and wall properties, Appl. Mathematics Comput. 263 (2015) 378-391

[10] M. Rehman, S. Noreen, A. Haider, H. Azam, Effect of heat sink/source on peristaltic flow of Jeffrey fluid through a symmetric channel, Alexandria Eng. J. 54 (2015) 733-743

[11] N.S. Akbar, Z.H. Khan, Heat transfer analysis of the peristaltic instinct of biviscosity fluid with the impact of thermal and velocity slips, Int. Commun. Heat Mass Transf. 58 (2014) 193-199

[12] M. Saleem, A. Haider, Heat and mass transfer on the peristaltic transport of non-Newtonian fluid with creeping flow, Int. J. Heat Mass Transf. 68 (2014) 514-526

[13] T.K. Hung, T.D. Brown, Solid-particle motion in twodimensional peristaltic flows, J. Fluid Mech. 73 (1976) $77-96$

[14] L.M. Srivastava, V.P. Srivastava, Peristaltic transport of a particle-fluid suspension, J. Biomech. Eng. 111 (1989) $157-165$

[15] J.C. Misra, S.K. Pandey, Peristaltic transport of a particle-fluid suspension in a cylindrical tube, Comput. Mathematics Appl. 28 (1994) 131-145

[16] K.S. Mekheimer, E.F. El Shehawey, A.M. Elaw, Peristaltic motion of a particle-fluid suspension in a planar channel, Int. J. Theoretical Phys. 37 (1998) 28952920

[17] P. Nagarani, G. Sarojamma, Peristaltic transport of small particles - power law fluid suspension in a channel, Australasian Phys. Eng. Sci. Medicine 30 (2007) 185-193

[18] J. Yao, K. Tao, Z. Huang, Flow of particulate-fluid suspension in a channel with porous walls, Transport in porous media 98 (2013) $147-172$

[19] M.M. Bhatti, A. Zeeshan, N. Ijaz, Slip effects and endoscopy analysis on blood flow of particle-fluid suspension induced by peristaltic wave, J. Molecul. Liquids 218 (2016) 240-245

[20] T. Hayat, O.U. Mehmood, Slip effects on MHD flow of third order fluid in a planar channel, Communications in Nonlinear Science and Numerical Simulation 16 (2011) 1363-1377

[21] A. Sinha, G.C. Shit, N.K. Ranjit, Peristaltic transport of MHD flow and heat transfer in an asymmetric channel: Effects of variable viscosity, velocity-slip and temperature jump, Alexandria Eng. J. 54 (2015) 691-704

[22] M. Hameed, A.A. Khan, R. Ellahi, M. Raza, Study of magnetic and heat transfer on the peristaltic transport of a fractional second grade fluid in a vertical tube, Eng. Sci. Technol. Int. J. 18 (2015) 496-502 
[23] S. Akram, S. Nadeem, A. Hussain, Effects of heat and mass transfer on peristaltic flow of a Bingham fluid in the presence of inclined magnetic field and channel with different wave forms, J. Magnetism Magnetic Mater. 362 (2014) 184-192

[24] R. Ellahi, M.M. Bhatti, A. Riaz, M. Sheikholeslami, Effects of magnetohydrodynamics on peristaltic flow of Jeffrey fluid in a rectangular duct through a porous medium, J. Porous Media 17 (2014) 143-157
[25] S. Hina, MHD peristaltic transport of Eyring-Powell fluid with heat/mass transfer, wall properties and slip conditions, J. Magnetism Magnetic Mater. 404 (2016) 148-158

[26] T. Hayat, E. Momoniat, F.M. Mahomed, Peristaltic MHD flow of third grade fluid with an endoscope and variable viscosity, J. Nonl. Mathematical Phys. 15 (2008) 91-104 